\title{
1 Mannsein als Risikofaktor
}

\author{
Lena Spangenberg und Elmar Brähler
}

Ist das Mannsein ein Risikofaktor für ein kürzeres Leben und für verschiedene Erkrankungen? Dieser Frage soll anhand epidemiologischer Daten in diesem Kapitel nachgegangen werden. Dazu werden verschiedene Aspekte wie Lebenserwartung und somatische und psychische Morbidität näher beleuchtet und diskutiert. Die Zielstellung ist dabei weniger, eine umfassende und erschöpfende Darstellung der Thematik zu liefern, sondern es soll mittels ausgesuchter Befunde und möglicher Erklärungsansätze ein Einblick gewonnen werden.

\subsection{Männer sterben früher}

In den letzten Jahren wurde der Gesundheit der Männer zunehmend mehr Beachtung geschenkt. Dies ist vor dem Hintergrund, dass männlich zu sein heutzutage in entwickelten Ländern den größten allein stehenden demografischen Risikofaktor für frühzeitige Sterblichkeit darstellt, durchaus gerechtfertigt (Kruger u. Nesse 2004). So steigt beispielsweise in Deutschland die Lebenserwartung seit Mitte des 19. Jahrhunderts an. Betrachtet man die vergangenen 50 Jahre (s. Tab. 1) wird jedoch deutlich, dass Frauen durchweg eine höhere Lebenserwartung hatten (Bundesministerium für Gesundheit 1997, 1999, 2001; Statistisches Bundesamt 2009).

Tab. 1 Lebenswartung für Männer und Frauen bei Geburt (in Jahren)

\begin{tabular}{|c|c|c|c|c|}
\hline & 1949/1951 & $1970 / 1972$ & 1988/1990 & $2006 / 2008$ \\
\hline \multicolumn{5}{|l|}{ West } \\
\hline Männer & 64,6 & 67,4 & 72,6 & 77,4 \\
\hline Frauen & 68,5 & 73,8 & 79,0 & 82,5 \\
\hline \multicolumn{5}{|l|}{ Ost } \\
\hline Männer & 65,1 & 69,0 & 70,0 & 76,1 \\
\hline Frauen & 69,1 & 73,7 & 76,2 & 82,2 \\
\hline
\end{tabular}


Anhand epidemiologischer Daten zu Mortalität und Morbidität im Geschlechtervergleich soll diskutiert werden, ob das Mannsein per se einen Risikofaktor darstellt oder ob noch mehr Faktoren in Betracht gezogen werden sollten, um diese Unterschiede in der Lebenserwartung zu erklären.

\subsubsection{Einflüsse auf die Lebenserwartung}

Die Entwicklung der Lebenserwartung in Deutschland (Ost/West) zwischen 1970 und 1990 demonstriert, wie sich gesellschaftliche Bedingungen auf die Lebenswartung auswirken können (s. Tab. 1). Seit der Wiedervereinigung schließt sich die Schere zwischen den alten und neuen Bundesländern allmählich wieder. Zwar haben Männer in den neuen Bundesländern nach wie vor eine geringere Lebenserwartung, allerdings finden sich mittlerweile vorwiegend ein Nord-Süd-Effekt sowie einige spezifische regionale Unterschiede. Die höhere Lebenserwartung in den „Speckgürteln“ in Süddeutschland lässt vermuten, dass die ökonomische Situation eine mögliche Erklärung liefert. Zwischen dem Armutsrisiko und der mittleren Lebenserwartung wurde auch ein Zusammenhang gefunden (Robert-Koch-Institut 2006; Leibniz-Institut für Länderkunde 2008).

Spannend ist in diesem Zusammenhang auch die Frage nach den Ursachen für die steigende Lebenserwartung. Unter anderem kann die Bildungsexpansion in Deutschland damit in Zusammenhang gebracht werden (Unger et al. 2009). Der Effekt der Höherqualifizierung kann sowohl direkt (Berufe mit weniger gesundheitlichen Belastungen) als auch indirekt sein (gesündere Lebensweise, kontinuierlichere Erwerbsverläufe).

\subsubsection{Beschwerdefreie Lebenserwartung}

Zwar scheinen Männer bisher im Vergleich zu Frauen eher kurz zu leben, dafür allerdings beschwerdefreier (Robert-Koch-Institut 2006). So leben beide Geschlechter im Durchschnitt bis zum Alter von etwa 63 Jahren beschwerdefrei. Zieht man in Betracht, dass Frauen im Mittel 5 Jahre länger leben, kann geschlussfolgert werden, dass diese Zeit mit Behinderungen und Beeinträchtigungen verbunden ist. Untermauert wird dies, wenn der Anteil der Pflegebedürftigen verglichen wird. Bei den 2 75-Jährigen ist der Anteil der weiblichen Pflegebedürftigen höher. Diese Differenz nimmt mit dem Alter zu und erreicht bei den über 9o-Jährigen das Maximum: 62,7\% der Frauen und 37,5\% der Männer sind in dieser Altersgruppe pflegebedürftig (RobertKoch-Institut 2004).

\subsubsection{Geschlechtsspezifische Mortalität}

Bereits im frühen Alter haben Jungen eine erhöhte Sterblichkeit (Bründel u. Hurrelmann 1999):

- Spontanabort häufiger bei männlichen Föten

- mehr Totgeburten (115:100)

- mehr Todesfälle im ersten Lebensjahr (498:403,8 je 100.ooo Lebendgeborene) 
In der Todesursachenstatistik für unter 65-Jährige weisen Männer in den Krankheitshauptgruppen im Vergleich zu Frauen höhere Mortalitätsziffern auf (Gesundheitsberichterstattung des Bundes 2009). Vergleichsgrundlage ist dabei das geschlechtsspezifische Mortalitätsverhältnis (Sterbefälle je 100.ooo Männer/Sterbefälle je 100.00o Frauen). Es beträgt bei Endokrinopathien 1,97 und bei Krankheiten des Atmungssystems 1,67. Unter den Erkrankungsgruppen, auf die mehr als dreiviertel der Todesfälle der unter 65-Jährigen zurückzuführen sind, gibt es auch deutliche Geschlechtsunterschiede (Neubildungen 1,29; Erkrankungen des Kreislaufssystems 2,71; Verletzungen und Vergiftungen 3,26; Erkrankungen des Verdauungssystems 2,19).

Des Weiteren ist die Zahl der Verkehrstoten bei Männern, trotz einer insgesamt sinkenden Zahl, nach wie vor zwei bis dreimal höher als bei Frauen (18,6 vs. 5,5 je 100.ooo Einwohner). Dies gilt im Besonderen für 15- bis 25-Jährige (Bundesministerium für Gesundheit 2008). Hinsichtlich der Suizidraten ist festzuhalten, dass sich eine deutlich geringere Zahl an Frauen das Leben nimmt; mehr als 75\% der Suizide werden von Männern verübt. Dabei finden sich besonders hohe Suizidraten in der der Gruppe der über 75-jährigen Männer (Möller-Leimkühler 20o8; Leibniz-Institut für Länderkunde 2007).

\subsection{Morbidität bei Männern und Frauen}

\subsubsection{Körperliche Erkrankungen}

Beurteilt man anhand ausgewählter körperlicher Krankheitsbilder, die aufgrund ihrer Häufigkeit, ihrer ökonomischen Folgen oder assoziierten Mortalität von Relevanz sind, den Gesundheitszustand von Männern und Frauen, wird schnell deutlich, dass im Bereich der somatischen Morbidität ebenfalls Geschlechtsunterschiede vorhanden sind. In Abbildung 1 ist die Prävalenz dieser bedeutsamen Erkrankungen dar-

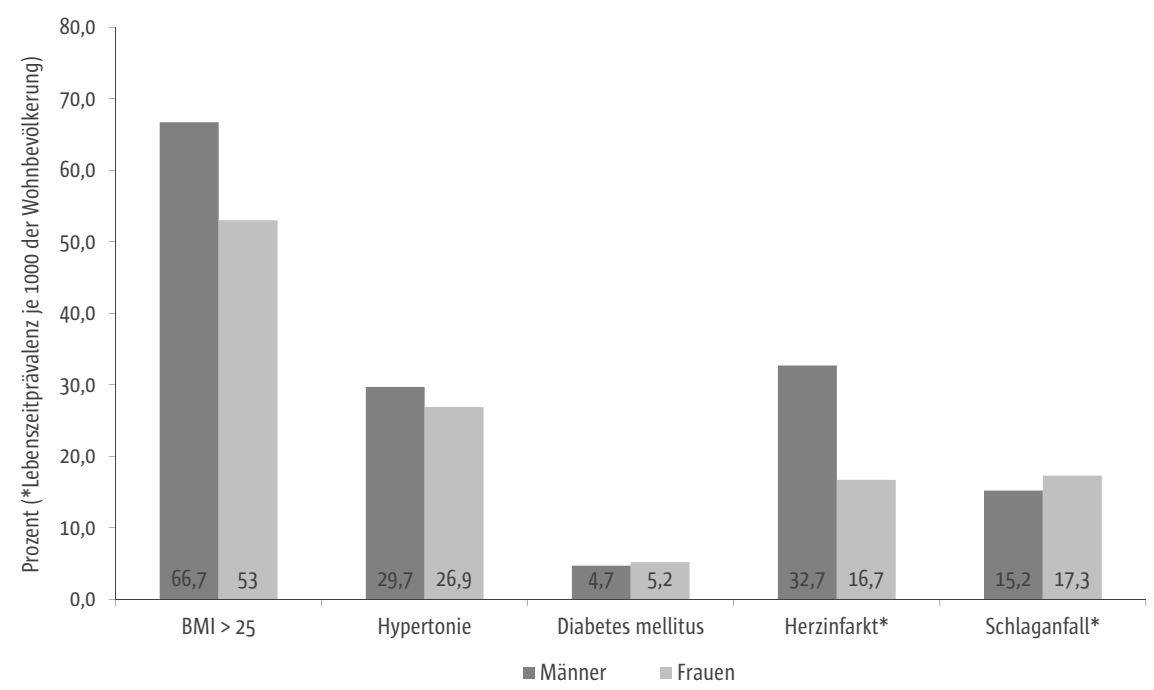

Abb. 1 Geschlechtsspezifische Prävalenz ausgesuchter Erkrankungen in der deutschen Bevölkerung (18 bis 79 Jahre) 
gestellt. Die für Deutschland repräsentativen Daten entstammen dem Bundesgesundheitssurvey 1998 (Robert-Koch-Institut 2006; Thamm 1999; Thefeld 2000; Thefeld 1999; Wiesner et al. 1999a; Wiesner et al. 1999b).

Bei dieser globalen Betrachtung über alle Altersgruppen zeigt sich, dass Männer häufiger Hypertonie haben, unter Übergewicht leiden (Body Mass Index [BMI, $\left.\mathrm{kg} / \mathrm{m}^{2}\right]>25$ ) und häufiger Herzinfarkte erleiden. Frauen hingegen zeigen tendenzielle höhere Werte bei den Diagnosen Diabetes mellitus und Schlaganfall. Zieht man weitere Faktoren in Betracht, wird deutlich, dass Morbiditätsunterschiede auch stets differenziert betrachtet werden sollten. Neben einer Zunahme der Morbidität bei allen angeführten Erkrankungen mit dem Alter treten Geschlechtsunterschiede zum Teil in manchen Altersgruppen nicht oder in anderer Richtung auf. Anhand einiger Befunde sei dies exemplarisch verdeutlicht: Die insgesamt höhere Prävalenz der Diabetes-Erkrankungen bei Frauen liegt in der sehr hohen Prävalenz bei den 70- bis 79-jährigen Frauen begründet (Thefeld 1999). Schlaganfälle sind bei den 50- bis 59-Jährigen Männern deutlich häufiger als bei Frauen, während diese in allen anderen Altersgruppen höhere Werte aufweisen (Wiesner 1999b). Auch regionale und soziale Einflüsse lassen sich finden. So sind beispielsweise Diabetes und Hypertonie in Ostdeutschland häufiger (Thefeld 1999; Thamm 1999). Je höher die soziale Schichtgehörigkeit ist, desto niedriger ist die Wahrscheinlichkeit übergewichtig zu sein oder Bluthochdruck zu haben (Thefeld 2000), umgekehrt verhält es sich mit Diabetes mellitus (Typ-II) (Robert-Koch-Institut 2006; Thefeld 1999). In Analysen zu Veränderungen des Krankheitsgeschehens zeigt sich zudem, dass Frauen in Bezug auf Herz-Kreislauf-Erkrankungen eine zunehmende Risikobelastung haben und die Inzidenz von Herzinfarkten bei 25bis 54-Jährigen ansteigend ist (Robert-Koch-Institut 2006; Thefeld 2000).

Morbiditätsunterschiede lassen sich auch in weiteren Krankheitsgruppen finden (Robert-Koch-Institut 2006): Chronische Rückenschmerzen, die infolge damit einhergehender Arbeitsunfähigkeit und Frühberentung von großer Relevanz sind, sind ähnlich wie andere Schmerzsymptome häufiger bei Frauen (22\%) als bei Männern $(15 \%)$. Als mögliche Gründe werden unterschiedliche Schmerz-Risikofaktoren oder Wahrnehmungsunterschiede diskutiert. Die Inzidenz von Krebserkrankungen wiederum lag bei Männern im Jahr 2002 mit 452 im Vergleich zu 335 bei Frauen je 100.00o Einwohner höher. Während Männer vor allem an Lungenkrebs erkranken, ist bei Frauen der Brustkrebs am häufigsten. Auch hier gibt es Veränderungen: So tritt bei Frauen Lungenkrebs infolge des angestiegenen Nikotinkonsums zunehmend häufiger auf.

\subsubsection{Psychische Störungen}

Im Bereich der psychischen Störungen finden sich bereits bei Kindern und Jugendlichen geschlechtsspezifische Unterschiede. Der Kinder- und Jugendgesundheitssurvey hat die Symptombelastung auf verschiedenen Dimensionen psychischer Gesundheit in einer bundesweiten repräsentativen Stichprobe (3 bis 17 Jahre) untersucht. $17,8 \%$ der Jungen und $11,5 \%$ der Mädchen können demnach als verhaltensauffällig oder grenzwertig verhaltensauffällig betrachtet werden. Bei dieser Zuordnung handelt es sich nicht um klinische Diagnosen (Hölling et al. 2007). Eine mögliche Erklärung für diesen Befund, dass Jungen eher dazu neigen, psychische Probleme zu externalisieren und damit eine vorhandene Problematik auch für das Umfeld leichter 
sichtbar ist, während Mädchen eher internalisieren (Rothenberger et al. 20o8). Eine Substichprobe 7- bis 17-Jähriger (BELLA-Studie) wurde in Hinblick auf psychische Probleme näher untersucht. In Tabelle 2 finden sich alters- und geschlechtsspezifische Prävalenzen ausgewählter Störungen (Ravens-Sieberer et al. 2008). Während Mädchen höhere Prävalenzen bei Angststörungen aufweisen, liegen Jungen bei Aufmerksamkeitsdefizit-/Hyperaktivitätsstörungen und Verhaltensstörungen vorn. Die Unterschiede bei Depression sind geringfügig.

Bei Erwachsen zeigt sich hinsichtlich der Prävalenz psychischer Störungen ein anderes Bild. Die Ergebnisse des Zusatzmoduls Psychische Störungen im Bundesgesundheitssurvey zeigen, dass mit Ausnahme der Substanzstörungen Frauen häufiger von psychischen Störungen betroffen sind als Männer. Dies gilt insbesondere bei affektiven und Angststörungen (Jacobi et al. 2004a; Jacobi et al. 2004b). Alters- und geschlechtsspezifische Prävalenzen für wichtige Diagnosegruppen (DSM-IV) sind in Tabelle 3 aufgeführt.

Allerdings wird vermutet, dass die gefundenen Geschlechtsunterschiede zumindest teilweise auch darin begründet liegen, dass frauentypische Störungen umfassender erfasst wurden. Eine andere Erklärung hinsichtlich der zumeist niedrigeren Präva-

Tab. 2 Prävalenz von Kindern und Jugendlichen mit psychischen Symptomen und Beeinträchtigung in ausgewählten Störungsgruppen (Zahlen aus Ravens-Sieberer et al. 2008)

\begin{tabular}{lcccc} 
& Depression (in \%) & Angst (in \%) & ADHS (in \%) & Verhaltensstörung (in \%) \\
\hline 7-10 lahre & & & & \\
\hline Mädchen & 5,6 & 7,5 & 2,2 & 6,7 \\
\hline Jungen & 5,5 & 5,2 & 5,5 & 10,5 \\
\hline 11-17 lahre & & & & 8,4 \\
\hline Mädchen & 4,6 & 4,2 & 1,5 & 10,9 \\
\hline Jungen & 5,3 & 3,8 & 2,8 & \\
\hline
\end{tabular}

Tab. 3 12-Monats-Prävalenz ausgesuchter psychischer Störungen bei Frauen und Männern (Zahlen aus Jacobi et al. 2004b)

\begin{tabular}{|c|c|c|c|c|c|c|c|c|}
\hline \multirow[t]{2}{*}{ Diagnosegruppe } & \multicolumn{2}{|c|}{ 18-34 Jahre (\%) } & \multicolumn{2}{|c|}{ 35-49 Jahre (\%) } & \multicolumn{2}{|c|}{ 50-65 Jahre (\%) } & \multicolumn{2}{|c|}{ Gesamt (\%) } \\
\hline & Frauen & Männer & Frauen & Männer & Frauen & Männer & Frauen & Männer \\
\hline Substanzstörungen* & 2,9 & 12,3 & 1,7 & 5,3 & 0,6 & 3,6 & 1,7 & 7,2 \\
\hline affektive Störungen & 13,4 & 9,4 & 16,8 & 8,5 & 15,9 & 7,6 & 15,4 & 8,5 \\
\hline Angststörungen & 20,0 & 8,0 & 19,4 & 10,0 & 19,9 & 9,7 & 19,8 & 9,2 \\
\hline $\begin{array}{l}\text { somatoforme } \\
\text { Störungen }\end{array}$ & 14,9 & 5,7 & 15,2 & 7,3 & 14,7 & 8,6 & 15,0 & 7,1 \\
\hline Essstörungen & 1,0 & 0,2 & 0,5 & 0,3 & 0,0 & 0,1 & 0,5 & 0,2 \\
\hline $\begin{array}{l}\text { irgendeine } \\
\text { psychische Störung }\end{array}$ & 38,0 & 27,4 & 36,5 & 25,9 & 36,5 & 22,2 & 37,0 & 25,3 \\
\hline
\end{tabular}

*Missbrauch und Abhängigkeit (ohne Nikotin) 
lenz depressiver Erkrankungen bei Männern ist, dass es eine „männliche“ Depression gibt, die durch die gängige Psychodiagnostik nicht erfasst wird. Angenommen wird, dass die erfragten depressiven Symptome als weiblich gelten und infolgedessen von Männern dissimuliert oder abgewehrt werden (Möller-Leimkühler 2008). Neben diesem deutlichen Geschlechtsunterschied, der sich auch in einer Metaanalyse europäischer Studien zeigte (Wittchen u. Jacobi 2005), ist festzuhalten, dass die Prävalenz psychischer Störungen auch regionalen Einflüssen zu unterliegen scheint: So sind einige psychische Störungen in den alten Bundesländern häufiger (Jacobi et al. 2004a), bei den affektiven Störungen blieb der Geschlechtsunterschied jedoch trotz der regionalen Unterschieds erhalten (Wittchen et al. 1999).

\section{Fazit}

Die anfänglich aufgeworfene Frage, ob das Mannsein ein Risiko für eine verkürzte Lebenserwartung und Erkrankungen bietet, lässt sich nicht global mit Ja oder Nein beantworten. Einige der hier referierten Befunde legen es nahe, andere wiederum nicht: Zwar ist die Lebenserwartung von Männern kürzer, allerdings spielen neben dem Geschlecht auch regionale und gesellschaftliche Faktoren eine Rolle. Es ist jedoch festzuhalten, dass die Mortalität von Männern bei vielen körperlichen Erkrankungen gegenüber Frauen erhöht ist, sie häufiger Opfer tödlicher Unfälle sind und höhere Suizidraten aufweisen als Frauen. Auch bei der somatischen Morbidität gibt es Geschlechtsunterschiede. Hier wird ebenfalls deutlich, dass nicht allein das Geschlecht, sondern auch Einflüsse wie soziale Schicht und Lebensstil eine Rolle spielen. Deutlich positiver hingegen fällt der Geschlechtervergleich hinsichtlich der Prävalenz psychischer Erkrankungen für die Männer aus. Obwohl im Kindes- und Jugendalter eher die Jungen zu Verhaltensauffälligkeiten neigen, sind im Erwachsenenalter Frauen deutlich häufiger von psychischen Störungen betroffen. Zusammenfassend lässt sich also sagen, dass es vielerlei Geschlechtsunterschiede gibt und diese häufig, aber nicht immer, negativ für die Männer ausfallen. Wie kommen diese Differenzen in Lebenserwartung, Mortalität und Morbidität nun zustande? Dazu gibt es verschiedene Erklärungsansätze.

So bietet die Frage, ob biologische oder soziale Faktoren die Differenzen bestimmen, ein äußerst vielschichtiges Forschungsfeld. In der sogenannten Klosterstudie wurde mittels einer Mortalitätsanalyse von mehr als 8.000 Nonnen und Mönchen aus Bayern untersucht, ob Sterblichkeitsunterschiede eher auf Biologie oder Verhalten zurückzuführen sind (Dinkel u. Luy 1999; Luy 2002). Aufgrund der klösterlichen Lebensweise konnten für die untersuchten Personen einige mortalitätsassoziierte Faktoren ausgeschlossen werden (Schwangerschaft, Säuglings- und Kindersterblichkeit, Unterschiede bei Gesundheit gefährdender Lebensführung, ungleiche Rollen von Ehepartnern), andere wie Rauch- oder Trinkverhalten bleiben jedoch schwer abzuschätzen. Es zeigte sich, dass Mönche älter werden als Männer der Allgemeinbevölkerung, während Nonnen im Vergleich zu Frauen aus der Allgemeinbevölkerung eine ähnlich hohe Lebenserwartung aufweisen. Allerdings ist die gefundene Differenz von zwei Jahren in der Lebenserwartung zwischen Mönchen und Nonnen trotz einer sich gleichenden Zunahme im letzten Jahrhundert stets konstant geblieben.

Sozialwissenschaftliche Fächer, die den Zusammenhang von Gender und Gesundheit erforschen, postulieren hingegen, dass Geschlechterrollen und -stereotypien ein unterschiedliches Gesundheitsverhalten zur Folge haben (Alfermann 1996; Courtenay 2000). So können bei Männern auch riskante Verhaltensweisen, ein anderer Umgang mit Beschwerden und eine andere Inanspruchnahme des Gesundheitssystems, die mit typischen Eigenschaften der männlichen Rolle zusammenhängen, Mortalität und Morbidität zweifelsohne (negativ) beeinflussen. 
Zufrieden stellende Erklärungen für Geschlechtsunterschiede wurden innerhalb dieser Erklärungsansätze bisher nur bedingt gefunden. Die Befunde haben zumeist eher spekulativen Charakter als eine empirisch gesicherte Basis. Eine biopsychosoziale Perspektive ist daher vonnöten. In Zukunft sollte die Forschung zu Geschlechtsunterschieden bei Lebenserwartung, Mortalität und Morbidität verschiedene biologisch-medizinische (wie genetische Dispositionen, physiologische und hormonelle Merkmale) und sozial-gesellschaftliche Faktoren (wie Bewältigungsstrategien, berufliche und familiäre Rollen) einbeziehen.

\section{Literatur}

Alfermann D (1996) Geschlechterrollen und geschlechtstypisches Verhalten. Kohlhammer, Stuttgart

Bründel H, Hurrelmann K (1999) Konkurrenz, Karriere, Kollaps. Männerforschung und der Abschied vom Mythos Mann. Kohlhammer, Stuttgart

Bundesministerium für Gesundheit (1997) Daten des Gesundheitswesens. Ausgabe 1997. Nomos, Baden-Baden

Bundesministerium für Gesundheit (1999) Daten des Gesundheitswesens. Ausgabe 1999. Nomos, Baden-Baden

Bundesministerium für Gesundheit (2001) Daten des Gesundheitswesens. Ausgabe 2001. Nomos, Baden-Baden

Bundesministerium für Gesundheit (2008) Daten des Gesundheitswesens. Ausgabe 2008. Nomos, Baden-Baden

Courtenay W (2000) Constructions of masculinity and their influence on men's well being: a theory of gender and health. Soc Sci Med 50, 1385-1401

Dinkel R, Luy M (1999) Natur oder Verhalten? Ein Beitrag zur Erklärung der männlichen Übersterblichkeit durch einen Vergleich von Kloster- und Allgemeinbevölkerung. Z Bevolkerungswiss 24, 105-132

Gesundheitsberichterstattung des Bundes (2009) Vorzeitige Sterblichkeit unter 65 lahren. http://www.gbe-bund. de/oowag21-install/servlet/oowa/aw92/dboowasys921.xwdevkit/xwd_init?gbe.isgbetol/xs_start_neu/ \&p_aid=3\&p_aid=3848577\&nummer=562\&p_sprache=D\&p_indsp=-\&p_aid=55650759, abgerufen am 19.10.2011

Hölling H, Erhart M; Ravens-Sieberer U, Schlack R (2007) Verhaltensauffälligkeiten bei Kindern und Jugendlichen. Erste Ergebnisse aus dem Kinder- und Jugendgesundheitssurvey (KiGGS). Bundesgesundheitsbl - Gesundheitsforsch - Gesundheitsschutz 50, 784-793

Jacobi F, Hoyer |, Wittchen HU (2004a) Mental health in East and West Germany: Analyses of the German National Health Interview and Examination Survey. Z Klin Psychol Psychother 33, 251-260

Jacobi F, Klose, M, Wittchen HU (2004b) Psychische Störungen in der Allgemeinbevölkerung: Inanspruchnahme von Gesundheitsleistungen und Ausfalltage. Bundesgesundheitsbl - Gesundheitsforsch - Gesundheitsschutz 47, 736-744

Kruger DI, Nesse RM (2004) Sexual selection and the Male:Female Mortality Ratio. Evolutionary Psycholog 2, 66-77

Leibniz-Institut für Länderkunde (2007) Suizide - Sterblichkeit 1980-2007 nach Geschlecht. http://aktuell. nationalatlas.de/Suizidsterbefaelle.1_01-2010.0.html, abgerufen am 15.02.2010

Leibniz-Institut für Länderkunde (2008). Lebenserwartung Lebendgeborener 2005 nach Raumordnungsregionen. http://aktuell.nationalatlas.de/Lebenserwartung.11_10-2008.0.html, abgerufen am 16.02.2010

Luy M (2002) Warum Frauen länger leben - Erkenntnisse aus einem Vergleich von Kloster- und Allgemeinbevölkerung. Materialien zur Bevölkerungswissenschaft 106, Bundesinstitut für Bevölkerungsforschung, Wiesbaden

Möller-Leimkühler A (2008) Depression - überdiagnostiziert bei Frauen, unterdiagnostiziert bei Männern? Gynäkologe 41, 381-388

Ravens-Sieberer U, Wille N, Erhart M, Bettge S, Wittchen HU, Rothenberger A, Herpertz-Dahlmann B, Resch F, Hölling H, Bullinger M, Barkmann C, Schulte-Markwort M, Döpfner M; BELLA study group (2008) Prevalence of mental health problems among children and adolescents in Germany: results of the BELLA study within the National Health Interview and Examination Survey. Eur Child Adolesc Psychiatry 17, 22-33

Robert-Koch-Instititut (Hrsg.) in Zusammenarbeit mit dem Statistischen Bundesamt (2006) Gesundheitsberichterstattung in Deutschland. Gesundheit in Deutschland. Robert-Koch-Institut, Berlin

Robert-Koch-Institut (2004) Gesundheitsberichterstattung des Bundes. Themenheft Pflege. Robert-Koch-Institut, Berlin 
Rothenberger A, Becker A, Erhart M, Wille N, Ravens-Sieberer U (2008) Psychometric properties of the parent strengths and difficulties questionnaire in the general population of German children and adolescents: results of the BELLA study. Eur Child Adolesc Psychiatry 17, 99-105

Statistisches Bundesamt (2009) Lebenserwartung. http://www.destatis.de/jetspeed/portal/cms/Sites/destatis/ Internet/DE/Navigation/Statistiken/Bevoelkerung/GeburtenSterbefaelle/Tabellen.psml, abgerufen am 14.02.2010

Thamm M (1999) Blutdruck in Deutschland - Zustandsbeschreibung und Trends. Gesundheitswesen 61, Sonderheft 2, 90-93

Thefeld W (1999) Prävalenz des Diabetes mellitus in der erwachsenen Bevölkerung Deutschlands. Gesundheitswesen 61, Sonderheft 2, 85-89

Thefeld W (2000) Verbreitung der Herz-Kreislauf-Risikofaktoren Hypercholesterinämie, Übergewicht, Hypertonie und Rauchen in der Bevölkerung. Bundesgesundheitsblatt Gesundheitsforschung Gesundheitsschutz 43, 415-423

Unger R, Schulze A, Klein T (2009) Educational expansion and life expectancy in Germany. In: Hadjar A, Becker R (Hrsg.) Expected and unexpected consequences of the educational expansion in Europe and the US. Bern, Haupt Verlag, 377-388

Wiesner G, Grimm J, Bittner E (1999a) Zum Herzinfarktgeschehen in der Bundesrepublik Deutschland: Prävalenz, Inzidenz, Trend, Ost-West-Vergleich. Gesundheitswesen 61, Sonderheft 2, 72-78

Wiesner G, Grimm I, Bittner E (1999b) Schlaganfall: Prävalenz, Inzidenz, Trend, Ost-West-Vergleich. Erste Ergebnisse aus dem Bundes-Gesundheitssurvey 1998. Gesundheitswesen 61, Sonderheft 2, 79-84

Wittchen HU, Jacobi F (2005) Size and burden of mental disorders in Europe - a critical review and appraisal of 27 studies. Eur Neuropsychopharmacol 15, 357-376

Wittchen HU, Müller N, Pfister H, Winter S, Schmidtkunz B (1999) Affektive, somatoforme und Angststörungen in Deutschland - Erste Ergebnisse des bundesweiten Zusatzsurveys „Psychische Störungen“. Gesundheitswesen 61, Sonderheft 2, 216-222 